\title{
Multiplication facts and the mental number line: evidence from unbounded number line estimation
}

\author{
Regina M. Reinert • Stefan Huber • \\ Hans-Christoph Nuerk · Korbinian Moeller
}

Received: 14 September 2013/Accepted: 13 December 2013/Published online: 3 January 2014

(C) Springer-Verlag Berlin Heidelberg 2014

\begin{abstract}
A spatial representation of number magnitude, aka the mental number line, is considered one of the basic numerical representations. One way to assess it is number line estimation (e.g., positioning 43 on a number line ranging from 0 to 100). Recently, a new unbounded version of the number line estimation task was suggested: without labeled endpoints but a predefined unit, which was argued to provide a purer measure of spatial numerical representations. To further investigate the processes determining estimation performance in the unbounded number line task, we used an adapted version with variable units other than 1 to evaluate influences of (i) the size of a given unit and (ii) multiples of the units as target numbers on participants' estimation pattern. We observed that estimations got faster and more accurate with increasing unit sizes. On the other hand, multiples of a predefined unit were estimated faster, but not more accurately than non-multiples. These results indicate an influence of multiplication fact knowledge on spatial numerical processing.
\end{abstract}

R. M. Reinert ( $\square)$

Center for Disability and Integration (CDI-HSG),

University of St. Gallen, Rosenbergstrasse 51,

9000 St. Gallen, Switzerland

e-mail: Regina.Reinert@unisg.ch

S. Huber $\cdot$ H.-C. Nuerk $\cdot$ K. Moeller

Knowledge Media Research Center,

Tüebingen, Germany

H.-C. Nuerk · K. Moeller

Department of Psychology, Eberhard-Karls University

Tüebingen, Tüebingen, Germany

\section{Introduction}

The mental representation of number magnitude is often described by referring to the metaphor of a mental number line. On this mental number line integers are supposed to be represented in ascending order according to their magnitude. A common task to assess the mental number line representation is the number line estimation task, in which participants have to estimate the position of a given number (e.g., 38) on an otherwise empty number line of a certain length specified by a starting and an endpoint (e.g., 0 to 100; e.g., Berteletti, Lucangeli, Piazza, Dehaene, \& Zorzi, 2010; Moeller, Pixner, Kaufmann, \& Nuerk, 2009a; Opfer \& Siegler, 2007; Siegler \& Opfer, 2003). From the observed estimation pattern it is then inferred on the nature of the underlying representation of number magnitude. Based on an evaluation of children's estimation patterns it has been claimed that children's mental number line representation develops from a logarithmically compressed to linear spacing with age and experience (e.g., Siegler \& Booth, 2004; Siegler \& Opfer, 2003; Booth \& Siegler, 2006). However, recently the validity of this conclusion was challenged. On the one hand, there is evidence indicating influences of counting capabilities (Ebersbach, Luwel, Frick, Onghena, \& Verschaffel, 2008) or place-value understanding (Moeller, Pixner, Kaufmann, \& Nuerk, 2009a, see also Helmreich, Zuber, Pixner, Kaufmann, Nuerk, \& Moeller, 2011; Moeller \& Nuerk, 2011a) to underlie children's performance development rather than a qualitative change in their mental number line representation.

On the other hand, there is accumulating evidence suggesting that inferences on the mental number line representation based on this commonly used bounded version of the number line estimation task should be taken with 
care. Instead of representing an isomorphic assessment of the mental number line representation performance in the bounded number line estimation task may be (also) determined by strategic processes (e.g., Barth \& Paladino, 2011; Slusser, Santiago, \& Barth, 2013). In particular, it was observed that participants not necessarily just estimated the position of a given number on an empty number line (e.g., 25 as 25 units to the right of 0 ), but referred to proportion judgment strategies inferring the position of a number by considering part-whole relations upon the number line by the use of external reference points (e.g., 25 is a quarter from 100 on a bounded number line from 0 to 100). This is corroborated by recent evaluations of the pattern of estimation errors in bounded number line tasks indicating estimations to be more accurate around reference points. For instance, when the two endpoints as well as the middle of a given number line serve as reference points this leads to a characteristic M-shaped error pattern with smaller and less variable estimation errors for target numbers around the reference points (i.e., 0, 50, and 100). In addition, this is reflected by estimation patterns described best by inverse sigmoid functions (e.g., Ashcraft \& Moore, 2012; Sullivan, Juhasz, Slattery \& Barth, 2011). Interestingly, such a view is consistent with predictions of psychophysical models of proportion judgment (e.g., Hollands \& Dyre, 2000; Spence, 1990). In sum, it is important to note that there is currently a controversial debate on the validity of conclusions on the nature of the mental number line representation based on data from the bounded number line estimation task (e.g., Barth \& Paladino, 2011; Sullivan, Juhasz, Slattery, \& Barth, 2011; Slusser et al., 2013).

Against this background, Cohen \& Blanc-Goldhammer (2011) suggested a new version of the number line estimation task that may overcome the limitations described above. Different from the bounded number line estimation task, their version of the number line estimation task had no labeled endpoint, and was therefore called an unbounded number line task. To allow for estimations of the position of a given target number on this unbounded number line, participants were also given the distance between 0 and 1 as the unit to base their estimations on (see also Booth \& Siegler, 2006 for a similar approach in children). In this way, participants then had to estimate the spatial position of target numbers ranging from 2 to 25 upon the given unbounded number line. Importantly, and in contrast to the characteristic M-shaped error pattern observed for the bounded number line task Cohen \& BlancGoldhammer (2011) found that error variability increased linearly with the size of the target numbers in their unbounded number line task. This corresponds nicely with models suggesting a linear layout of the mental number line (e.g., Gallistel \& Gelman, 1992). However, most importantly, the study of Cohen \& Blanc-Goldhammer (2011) suggests that this unbounded number line estimation task reflects a more unbiased measure of the mental number line representation as compared to the bounded number line estimation task. Nevertheless, even in the unbounded number line estimation task participants' estimations reflected specific consistencies. In particular, the authors observed that the participants adopted a "dead-reckoning" strategy to complete the unbounded number line task. The estimation pattern of this dead-reckoning strategy reflects repetitive scallops resulting when participants use multiples of a certain quantity, about 10 in the data of Cohen \& Blanc-Goldhammer (2011), as internal orientations for their estimations. Cohen \& Blanc-Goldhammer (2011) termed this range the participants' actual "working window of numbers" (p. 335). The authors suggest that when participants have to estimate the position of numbers above their working window they count to the last number in their working window and then start counting again, which results in the scalloped estimation pattern observed. For example, if participants had to indicate the position of 25 , they estimated 20 by counting to the end of their working window two times followed by counting to the half of the working window. Interestingly, this indicates that participants build up and use internal references to come to their estimations that reflects "steps" upon the number line, which are considerably larger than the predefined unit. This seems to suggest that larger steps may facilitate estimation performance. In fact, $92 \%$ of the participants in Experiment 2 of Cohen \& Blanc-Goldhammer (2011) exhibited an estimation pattern that was fitted best by a scallop model. However, the results also suggest that the working windows differed between participants as there was evidence for single-, dual-, and multi-scallop strategies. From this, at least three questions arise.

First, it would be interesting to evaluate whether it is indeed the case that larger steps facilitate estimation performance. Based on the results of Cohen \& Blanc-Goldhammer (2011) one might speculate that the summed estimation error of, for instance, 2 scallops of about 10 is smaller and thus the estimation is more accurate as compared to the summed estimation error for 20 steps corresponding to a predefined unit of 1 . In the latter case, the summed estimation error may just be higher because of the accumulation due to the higher number of individual steps (each associated with a certain error).

Second, the fact that the working window differed between participants raises the question in how far the participants' working window can be influenced. While Cohen \& Blanc-Goldhammer (2011) used the unit 1 only, one might think of using different units (e.g., 2, 5, 10). In line with above hypothesis estimation performance should 
improve with increasing unit size as fewer steps are needed to reach the target numbers-just like in the case of scalloped internal references. However, this might be determined by either the fact that unit size approaches the average working window of about 10 or that the working window is adapted to the respective unit.

Finally, when it is indeed the case that participants benefit from relatively larger scallops/units one might hypothesize that multiples of the scallop/unit are estimated more accurately than non-multiples because no further localization is needed. A special role for multiples in numerical cognition is documented by a large body of research on the representation of multiplication facts. For instance, Rusconi, Galfano, Speriani, \& Umiltà (2004) observed that a multiplication product (e.g., 15) is automatically activated by the presentation of its two operands (e.g., 3 and 5). With respect to multiplication fact knowledge and the mental number line, the results of Nuerk, Geppert, van Herten, \& Willmes (2002) for a verification version of the number bisection task (NBT) are informative. The authors found that the decision whether or not the central number of a triplet represents the numerical middle of the outer two numbers (e.g., 18_21_24 vs. 18_20_24) was taken faster and more accurately when the triplet was part of multiplication table (e.g. 18_21_24) than for triplets that were not (e.g. 19_22_25). As each triplet of the NBT can be interpreted as a segment of the mental number line these data provide first evidence indicating that multiples of a number may also be represented prominently upon the mental number as compared to non-related numbers (see also Wood, Nuerk, Moeller, Geppert, Schnitker, Weber, \& Willmes, 2008; Moeller, Fischer, Nuerk, \& Willmes, 2009b; Moeller, Klein, Fischer, Nuerk, \& Willmes, 2011b for influences of multiplicativity in the NBT). With regard to the present study, these findings imply that multiples of a given unit should also be estimated faster than non-multiples. Yet, as regards estimation accuracy predictions are less clear. On the one hand, speed and estimation accuracy may be associated predicting multiples of the units to be estimated more accurately as well. On the other hand, current models of multiplication fact knowledge do not include any spatial aspect at all. Rather, it is assumed that multiplication fact knowledge is represented in verbally mediated format (e.g., Dehaene, Piazza, Pinel, \& Cohen, 2003; Lee \& Kang, 2002; Moeller, Klein, Fischer, Nuerk, \& Willmes, 2011b). In this case, no beneficial influence on estimation performance would be expected.

Taken together, the present study aimed at evaluating the processes underlying the dead-reckoning strategy in the unbounded number line estimation task as proposed by Cohen \& Blanc-Goldhammer (2011). Therefore, we used an adapted version of the unbounded number line estimation task as compared to Cohen \& Blanc-Goldhammer (2011): in accordance with above hypothesis regarding the influence of the size of the predefined unit on participants' (scalloped) estimation pattern we varied unit size systematically from 1 to 10 , whereas Cohen \& Blanc-Goldhammer (2011) only used a unit size of 1 . As the scalloped estimation pattern indicates an estimation strategy referencing multiples of the unit sizes (about 10 times the unit 1 in Cohen \& Blanc-Goldhammer, 2011), manipulating unit size should influence the pattern of estimation errors significantly. In particular, our hypotheses were as follows: First, we expected that estimation performance should improve as the unit size increases. Second, we expected participants' working window of numbers to be influenced by unit size. Finally, we hypothesized target numbers that are multiples of the unit size to be estimated at least faster than non-multiples and possibly more accurately. To be able to evaluate these specific hypotheses, we extended the range of target numbers from 25 to 49 allowing for a systematic evaluation of unit sizes $2-10$ on estimation performance with particular interest being paid to multiples of the respective units.

\section{Method}

\section{Participants}

Twenty-seven students (7 male) of the University of Tübingen participated in the study in exchange for course credit. Mean age was 23.6 years with a standard deviation (SD) of 4.1 years (range 19-37 years). All participants reported normal or corrected-to-normal vision.

\section{Stimuli and design}

The experiment consisted of an unbounded number line estimation tasks, in which participants had to mark the position of a given target number on a number line by inferring the position of the target number from the length of a predefined unit. Generally, unbounded number lines were horizontal lines with lengths of 54, 58, 62 and 66 units randomly varying. Their physical length varied from 930 to 1,276 pixels with physical and numerical length uncorrelated to distract participants from generating expectations on the length of the unbounded number line. The target number was presented above the unit on the left end of the number line and surrounded by a circle. Both number line as well as target numbers and the respective unit were displayed in black color against a white background (see Fig. 1). 


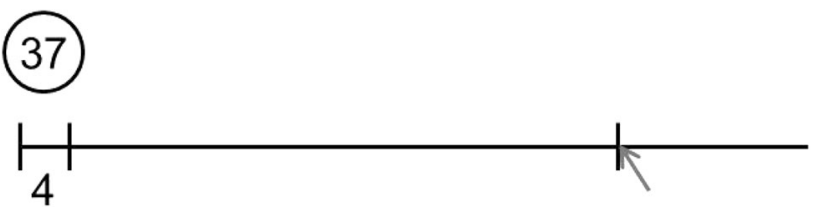

Fig. 1 Unbounded number line with variable unit

Importantly, we used an unbounded number line task with variable units ranging from 1 to 10 to indicate the reference for participants' estimations (see Fig. 1). We manipulated the size of the units to evaluate the deadreckoning strategy proposed by Cohen \& Blanc-Goldhammer (2011). The stimulus set of the unbounded number line task with variable units included selected two-digit numbers ranging from 11 to 49 as shown in the "Appendix". Target numbers were chosen to have the same mean problem size for all units, with regard to both overall number range as well as numbers within each decade (i.e., 11-19, 21-29, etc.). In addition, we manipulated whether target numbers were multiples or non-multiples of their respective unit size. Mean problem size was matched and it was aimed at matching the number of multiples and nonmultiples for each unit as far as possible. Twenty target numbers were presented for each unit reflecting five targets per decade and summing up to a total of 200 critical trials.

Stimuli were presented as pictures with a resolution of $1,920 \times 1,200$ pixels on a 26 " monitor. To prevent participants from using external reference points (e.g., the center of the screen or the position of the left edge of the screen), the location of the number line on the screen was chosen randomly for each trial, with the constraint that the number line did not protrude an area 200 pixels wide at all four edges of the monitor. In addition, the frame of the screen was covered by white tape to conceal possible landmarks used as external reference points.

\section{Procedure}

The experiment took place in a dimly lit room where participants were tested individually. Participants were seated approximately $60 \mathrm{~cm}$ away from the screen. Instructions focused on speed and accuracy and required participants to indicate the spatial position of the target number on the number line by a mouse click at their estimated position. Participants were not informed about the number range covered by the number line estimation task prior to completing it. Before each trial, a fixation mark appeared at the location of the screen where the origin of the number line was to be presented together with the mouse cursor. The number line and the to-be-estimated target number remained on the screen until a response was

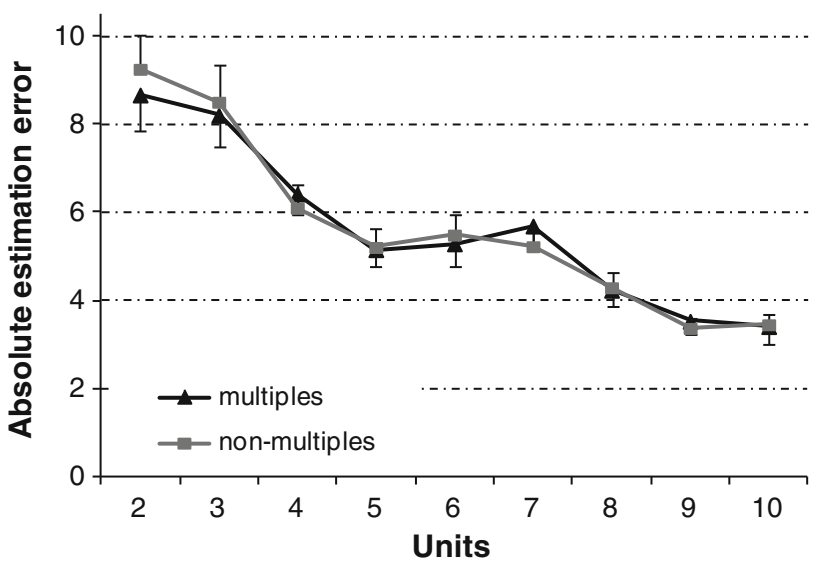

Fig. 2 Absolute estimation error for all units, error bars indicate 1 standard error of the mean (SEM)

given by a mouse click, directly followed by the fixation mark for the next trial. Items were presented in ten blocks with unit (i.e., 1-10) held constant within each block and block order randomized for each participant individually. In sum, the experiment took approximately $20 \mathrm{~min}$.

\section{Results}

Both absolute estimation error as the deviation of estimated position from the actual position of the respective target number on the unbounded number line as well as reaction times (RT) were analyzed. The data of one participant had to be excluded from the analysis due to individual mean RT being more than three SD longer as compared to mean RT over all participants.

\section{Absolute estimation error}

In a first step, mean estimates were calculated for each participant, separate for multiples and non-multiples of all units from 2 to 10 (unit 1 was excluded from further analyses as the distinction between multiples and nonmultiples is not possible). Then, we computed the absolute estimation error for all units (see Fig. 2). To investigate the influence of a multiplicative between target numbers and units, a $2 \times 9$ repeated-measures ANOVA with the factors multiplicativity (multiples vs. non-multiples) and units (2, $3,4,5,6,7,8,9$ vs. 10) was conducted. Evaluating whether multiplicative targets were localized more accurately than non-multiplicative revealed that the mean estimation error was not reliably smaller for multiplicative as compared to non-multiplicative targets $\left[M_{\text {Multiples }}=5.62\right.$ vs. $M_{\text {Non-mul- }}$ tiples $\left.=5.65 ; F(1,25)<1, \eta_{p}^{2}=0.003\right]$. In contrast, the main effect of units was significant $F(8,200)=22.58$, 


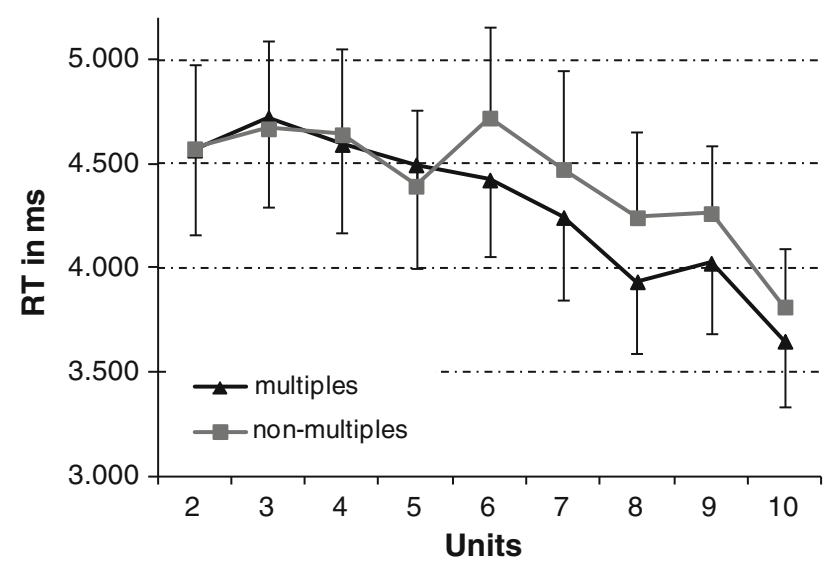

Fig. 3 Mean reaction times for the estimations of multiples and nonmultiples, error bars indicate 1 SEM

$p<0.001, \eta_{p}^{2}=0.475, \mathrm{GG}=0.473$, indicating that the absolute estimation errors differed between units. Most importantly for our hypothesis, however, contrast analyses indicated a significant linear trend for the estimation error over the units $F(1,25)=65.64, p<0.001, \eta_{p}^{2}=0.724$, indicating that the estimation error decreased with increasing unit size: the larger the unit, the more accurate the estimations of the target number on the number line. Finally, the interaction of multiplicativity and the units was not significant $\left.F(8,200)<1, \eta_{p}^{2}=0.031\right]^{1}$.

\section{Response latencies}

In a second step, RTs were also submitted to an identical $2 \times 9$ repeated-measures ANOVA discerning the factors multiplicativity and units (mean RT for all units are shown in Fig. 3). The main effect of multiplicativity was significant $F(1,25)=5.48, p=0.027, \eta_{p}^{2}=0.180$, indicating that targets that were multiples of a unit were estimated faster ( $\left.M_{\text {Multiples }}=4,295 \mathrm{~ms}\right)$ as compared to non-multiples $\left(M_{\text {Non-multiples }}=4,421 \mathrm{~ms}\right)$. In addition, the main effect of units was significant $F(8,200)=3.01, \quad p=0.013$, $\eta_{p}^{2}=0.107, \mathrm{GG}=0.629$, meaning that there were reliable differences in the response latencies between units. Comparable to the analysis of estimation errors, we also observed a significant linear trend $F(1,25)=12.02, p=0.002$, $\eta_{p}^{2}=0.325$ for $\mathrm{RT}$ over the units indicating that $\mathrm{RT}$ decreased with increasing unit size. At last, the interaction of multiplicativity and units was not significant for RT $F(8$, 200) $=0.97, p=0.415, \eta_{p}^{2}=0.037, \mathrm{GG}=0.388$. However, inspection of Fig. 3 indicates that the advantage for

\footnotetext{
${ }^{1}$ Please note that the results did not change substantially when using the absolute estimation error in pixels (instead of the relative measure of units) as the dependent variable.
}

multiples is mainly driven by reactions for units larger than 5. This was corroborated by an additional ANOVA discerning the factors multiplicativity and unit ( $\leq 5$ vs. $>5$ ) revealing a marginally significant interaction of small and large unit sizes $\left[F(1,25)=3.65, p=0.068, \eta_{p}^{2}=0.127\right]$ with a tendency for an RT advantage of multiples with unit sizes larger than 5 ( $247 \mathrm{~ms}$ vs. $-26 \mathrm{~ms}$ ).

\section{Working window}

Sizes of individual working windows were estimated applying the multi-scallop model of Cohen \& BlancGoldhammer (2011). This model is specified by two free parameters: the specific exponent and the size of the working window (see Cohen \& Blanc-Goldhammer, 2011, for a mathematical description of the model). Parameter estimates for each unit and each participant were obtained using the trust region-fitting algorithm implemented in Matlab 7.14 (MathWorks ${ }^{\circledR}$ ). Sizes of working windows were then compared conducting a repeated-measures ANOVA with the factor units. The main effect of units was not significant indicating that the size of the working window was not influenced by the size of the units [overall $M=9.52, \mathrm{SD}=0.31 ;$ range $9.11-10.07, F(8,200)<1$, $\left.\eta_{p}^{2}=0.024\right]$.

Taken together, in line with our hypotheses we observed that multiples of the respective units were estimated faster, but not more accurately than non-multiples and that participants' estimates indeed became more accurate and faster with increasing unit size. However, in contrast to our hypothesis, participants' working windows were not influenced by unit size.

\section{Discussion}

The present study was set up to evaluate the processes underlying the dead-reckoning strategy in the unbounded number line task as proposed by Cohen \& Blanc-Goldhammer (2011) using an adapted version of their task with variable unit sizes. We hypothesized that (1) estimation performance should improve with increasing unit size. Furthermore, (2) we expected that participants' working window of numbers would be influenced by unit size, and that (3) target numbers which are multiples of the unit size should be estimated at least faster than non-multiples. In the following, we will discuss these issues in turn.

The number of steps taken on the number line

In line with our first hypothesis, we observed that participants' estimates indeed became faster and more accurate 
with increasing unit size. We observed linear trends over the units for both RT and estimation errors indicating that the larger the unit the faster and more accurate the estimations of the target number on the number line. As hypothesized in the introduction such a result pattern may be driven by the number of steps (in terms of multiples of the unit) necessary to come to the target number. The more individual steps are to be taken the higher the overall estimation error for the sum of these steps accumulates due to the repeated error for each individual step. Consider, for instance, the accumulation of estimation error over 24 steps corresponding to a predefined unit of 2 to come to the target number 48 as compared to the same target number, but only 6 steps with a unit size of 8 . At the same time, higher accuracy was associated with shorter RTs as the participants needed fewer steps on the number line. Thus, larger unit sizes seemed to facilitate performance regarding both speed and accuracy. Taken together, these findings indicate that participants' performance was influenced reliably by unit size, and higher unit sizes were associated with better performance in the unbounded number line task.

\section{The working window of numbers}

Importantly, this is in line with the observation that the working window of numbers, i.e., the scallops to be taken by participants seemed to be independent of unit size. Against our expectations, participants' working window was not affected by the varying unit sizes, so that the size of this working window seemed to be fixed. Consistent with Cohen \& Blanc-Goldhammer (2011) reporting a working window of 10.6 for the single unit 1, we observed that the working window did not differ reliably between units 2 to 10 . This finding suggests that participants did not adapt the size of their working window to the respective unit, such as using scallops of 4 when they were presented with the unit size 4 . Instead, we observed that they seemed to use scallops of about 10 for their estimates ${ }^{2}$. Interestingly, above discussed data for RT and estimation accuracy provided converging evidence for the use of a working window of about 10 . With unit size approaching the size of the working window participants' estimates got faster and

\footnotetext{
2 Please note that the constant working window of about 10 we observed was not driven by the fact that 10 was the largest unit in the experiment. As recommended by Marc Brysbaert we conducted a control experiment in which 46 participants performed an unbounded number line estimation task with units 7 and 13 in a paper-pencil version of the task. Importantly, statistical evaluation of the resulting working windows indicated that it did not differ between unit size 7 and 13 [9.41 vs. 9.76 , respectively, $t(45)=1.07, p=0.32)]$ as well as from the mean working window over all unit sizes observed in the main experiment [unit size 7: $t(45)=0.43, p=0.67$; unit size 13 : $t(45)=0.84, p=0.41]$.
}

more accurate. More generally, this size of the working window complies quite nicely with the base-10 place-value structure of the Arabic number system.

While it has long been argued that the base-10 placevalue structure influences children's acquisition of counting abilities considerably (e.g., by special names for multiples of ten in most European languages, etc., cf. Price, 2001, see also Fuson, 1988, for a review), there is more recent evidence that base-10 place-value characteristics also influence other numerical tasks such as magnitude comparison (e.g., Nuerk, Weger, \& Willmes, 2001, see Nuerk, Moeller, Klein, Willmes, \& Fischer, 2011 for a review) and also number line estimation. Moeller, Pixner, Kaufmann, \& Nuerk, (2009a) observed that first graders' estimates in a bounded number line estimation task indicated initially separate representations of single- and twodigit numbers with a breakpoint at 10 . Moreover, Helmreich et al. (2011) found this to be language invariant at least for German- and Italian-speaking children. Nevertheless, the authors observed reliable effects of the way the place-value structure is conveyed in number words (i.e., the inversion principle in German number words, e.g., $24->$ vierundzwanzig literally translating to four and twenty) on children's number line estimation accuracy. Germanspeaking children for whom place-value ordering is inconsistent between verbal and symbolic Arabic notation were less accurate in their estimates. Synced with the present findings of a fixed working window of about 10 , these findings emphasize the important influence of the place-value structure of the Arabic number system on numerical cognition in general and number line estimation in particular.

The representation of multiples on the mental number line

With respect to the third hypothesis, we observed that multiplicativity had a significant influence on the performance in our unbounded number line estimation task: multiples of the respective units were estimated faster, but not more accurately than non-multiples. This indicates that in terms of steps upon the mental number line (see above), the location of multiples is reached faster possibly because there is no need to follow-up the initial estimate of a whole number of steps (i.e., multiples of the unit) by adjustments to either the left or right which are needed in the case of non-multiples of the units. Nevertheless, it is important to note that these final adjustments did not influence the estimation accuracy of non-multiples of the units, but only increased RT. Thus, the final adjustments seem to be an additional processing step amended at identical accuracy.

Interestingly, the current results further expand the observation of Nuerk, Geppert, van Herten, \& Willmes 
(2002; see also Moeller, Fischer, Nuerk, \& Willmes, 2009b; Moeller, Klein, Fischer, Nuerk, \& Willmes, 2011b) who found a facilitating effect of multiplicativity on participants' performance in a number bisection task also drawing heavily on the mental number line representation. Additionally, in line with our observation that the RT advantage for multiples was particularly driven by the relatively larger units Nuerk, Geppert, van Herten, \& Willmes (2002) found that the beneficial effect of multiplicatively related triplets was most pronounced when those triplets spanned a wide range upon the mental number line (e.g., 32_40_48 vs. 38_40_42). In sum, this seems to indicate that the benefits of multiplication fact knowledge are most prominent in those cases in which the standard procedure (i.e., magnitude manipulations in the NBT and number estimation in the current task) become more difficult, and thus error prone. However, it is important to note that neither in the NBT nor in the unbounded number line task intentional access to and/or active production of multiplication facts is required (cf. Moeller, Klein, Fischer, Nuerk, \& Willmes, 2011b for the NBT). Thus, as argued by Nuerk, Geppert, van Herten, \& Willmes (2002), for the NBT the observed influence of multiplication fact knowledge in the unbounded number line task indicates that it is not only the (spatial) representation of number magnitude which is assessed by this task. Instead, the advantage for multiples of a unit suggests that the unbounded number line task also recruits multiplication fact knowledge, and thus verbal numerical representations. However, in line with the assumption that multiplication fact knowledge is not represented spatially, we observed a reliable influence of multiplication fact knowledge on estimation speed but not accuracy-indicating that it may be related to the mental number line only indirectly.

Importantly, these results are also informative from a theoretical point of view. The currently most influential model of number processing - the Triple Code Model (TCM) by Dehaene and colleagues (Dehaene \& Cohen, 1995, 1997; Dehaene, Piazza, Pinel, \& Cohen, 2003) differentiates three representational codes underlying numerical cognition: (i) a visual-Arabic code allowing for the identification of digits as informative symbols, (ii) an analogue magnitude code representing the magnitude information associated with a specific number, and (iii) an auditory verbal code associated with number words but also arithmetic fact knowledge. Interestingly, the TCM suggests that these representational codes are task-specific in a sense that specific tasks are specifically associated with only one particular representational code (e.g., magnitude comparison-analogue magnitude code, multiplication-verbal code). Considering this, the present data provide additional evidence for the argument that the three representational codes may not be as task-specific as originally assumed in the TCM (Dehaene \& Cohen, 1995, 1997; Dehaene, Piazza, Pinel, \& Cohen, 2003). Instead, more than one representation seems to be recruited (i.e., the analogue magnitude as well as the verbal representation of a number in the present case; see also Nuerk et al., 2002; Wood, Nuerk, Moeller, Geppert, Schnitker, Weber, \& Willmes, 2008 for a more detailed discussion of this point). Finally, it needs to be acknowledged that these data are the first to indicate a recruitment of verbal numerical representations in number line estimation in adults. So far, there are only data on the influence of verbal representations on children's number line estimation performance (e.g., Helmreich et al., 2011). Thereby, there is now growing evidence for the notion that numerical cognition is an interactive process drawing on and adaptively integrating different kinds of representations and operations.

\section{Conclusions}

The present study set off to further investigate the influence of varying unit sizes on participants' estimation performance in a new unbounded number line estimation task proposed by Cohen \& Blanc-Goldhammer (2011). In particular, we were interested in whether (i) participants' performance was associated with unit size, (ii) unit size influences their working window of numbers, and (iii) multiples of the units were processed faster but not more accurately. The present results were meaningful on all these issues: we observed that participants' estimations improved with increasing unit size, possibly due to the fact that fewer steps reduced the accumulation of the estimation error. In line with this, we found that participants' working window was not influenced by unit size but seemed fix at about 10 for all unit sizes, suggesting that participants estimations got faster and more accurate as unit size approached their working window. Finally, extending previous findings on the effect of multiplication fact knowledge on processing spatial aspects of number magnitude in a number bisection task (e.g., Nuerk et al., 2002), we observed that multiples of a given unit benefited from the multiplication fact knowledge in terms of faster but not more accurate estimations. Thereby, the current data provide first evidence of a recruitment of verbal numerical representations in adults' number line estimations adding to similar findings in children and arguing for a task inclusive recruitment of numerical representations. 


\section{Appendix}

\begin{tabular}{|c|c|c|c|c|c|c|c|c|c|c|}
\hline Unit & 1 & 2 & 3 & 4 & 5 & 6 & 7 & 8 & 9 & 10 \\
\hline \multirow[t]{5}{*}{ Tens } & 11 & 12 & 12 & 12 & 11 & 12 & 12 & 12 & 13 & 11 \\
\hline & 12 & 13 & 14 & 13 & 13 & 13 & 13 & 13 & 14 & 13 \\
\hline & 16 & 16 & 16 & 16 & 15 & 14 & 14 & 16 & 15 & 15 \\
\hline & 18 & 18 & 17 & 17 & 18 & 18 & 18 & 17 & 16 & 18 \\
\hline & 19 & 19 & 18 & 18 & 19 & 19 & 19 & 18 & 18 & 19 \\
\hline \multirow[t]{5}{*}{ Twenties } & 21 & 20 & 20 & 20 & 20 & 21 & 20 & 21 & 21 & 20 \\
\hline & 23 & 21 & 21 & 23 & 22 & 23 & 21 & 23 & 22 & 23 \\
\hline & 24 & 24 & 25 & 24 & 25 & 24 & 23 & 24 & 23 & 25 \\
\hline & 26 & 27 & 27 & 26 & 26 & 25 & 28 & 26 & 27 & 26 \\
\hline & 27 & 28 & 29 & 28 & 28 & 28 & 29 & 27 & 28 & 27 \\
\hline \multirow[t]{5}{*}{ Thirties } & 32 & 30 & 30 & 32 & 30 & 30 & 31 & 32 & 31 & 30 \\
\hline & 34 & 34 & 32 & 33 & 34 & 34 & 33 & 33 & 32 & 33 \\
\hline & 35 & 35 & 36 & 36 & 35 & 36 & 35 & 35 & 36 & 36 \\
\hline & 36 & 37 & 38 & 37 & 38 & 37 & 38 & 37 & 38 & 38 \\
\hline & 39 & 39 & 39 & 38 & 39 & 39 & 39 & 39 & 39 & 39 \\
\hline \multirow[t]{5}{*}{ Forties } & 41 & 40 & 40 & 40 & 40 & 41 & 41 & 40 & 40 & 40 \\
\hline & 43 & 41 & 42 & 41 & 43 & 42 & 42 & 42 & 42 & 41 \\
\hline & 45 & 45 & 46 & 44 & 45 & 44 & 44 & 43 & 45 & 44 \\
\hline & 46 & 48 & 47 & 48 & 46 & 47 & 46 & 48 & 47 & 48 \\
\hline & 47 & 49 & 48 & 49 & 48 & 48 & 49 & 49 & 48 & 49 \\
\hline
\end{tabular}

Please note that multiples are indicated in italics

\section{References}

Ashcraft, M. H., \& Moore, A. M. (2012). Cognitive processes of numerical estimation in children. Journal of Experimental Child Psychology, 111, 246-267.

Barth, H. C., \& Paladino, A. M. (2011). The development of numerical estimation: evidence against a representational shift. Developmental Science, 14, 125-135.

Berteletti, I., Lucangeli, D., Piazza, M., Dehaene, S., \& Zorzi, M. (2010). Numerical estimation in preschoolers. Developmental Psychology, 46, 545-551.

Booth, J. L., \& Siegler, R. S. (2006). Developmental and individual differences in pure numerical estimation. Developmental Psychology, 41, 189-201.

Cohen, D. J., \& Blanc-Goldhammer, D. (2011). Numerical bias in bounded and unbounded number line tasks. Psychonomic Bulletin and Review, 18, 331-338.

Dehaene, S., \& Cohen, L. (1995). Towards an anatomical and functional model of number processing. Mathematical Cognition, 1, 83-120.

Dehaene, S., \& Cohen, L. (1997). Cerebral pathways for calculation: double dissociation between rote verbal and quantitative knowledge of arithmetic. Cortex, 33, 219-250.

Dehaene, S., Piazza, M., Pinel, P., \& Cohen, L. (2003). Three parietal circuits for number processing. Cognitive Neuropsychology, 20, 487-506.
Ebersbach, M., Luwel, K., Frick, A., Onghena, P., \& Verschaffel, L. (2008). The relationship between the shape of the mental number line and familiarity with numbers in 5- to 9-year old children: evidence for a segmented linear model. Journal of Experimental Child Psychology, 99, 1-17.

Fuson, K. (1988). Children's counting and concepts of cumber. New York: Springer.

Gallistel, C. R., \& Gelman, R. (1992). Preverbal and verbal counting and computation. Cognition, 44, 43-74.

Helmreich, I., Zuber, J., Pixner, S., Kaufmann, L., Nuerk, H.-C., \& Moeller, K. (2011). Language effects on children's mental number line: how cross-cultural differences in number word systems affect spatial mappings of numbers in a non-verbal task. Journal of Cross-Cultural Psychology, 42, 598-613.

Hollands, J. G., \& Dyre, B. P. (2000). Bias in proportion judgments: the cyclical power model. Psychological Review, 107, 500-524.

Lee, K. M., \& Kang, S. Y. (2002). Arithmetic operation and working memory: differential suppression in dual tasks. Cognition, 83, B63-B68.

Moeller, K., Fischer, M. H., Nuerk, H.-C., \& Willmes, K. (2009a). Eye fixation behaviour in the number bisection task: evidence for temporal specificity. Acta Psychologica, 131, 209-220.

Moeller, K., Klein, E., Fischer, M. H., Nuerk H.-C., \& Willmes, K. (2011b). Representation of multiplication facts: evidence for partial verbal coding. Behavioral and Brain Functions, 7:25.

Moeller, K., \& Nuerk, H.-C. (2011). Psychophysics of numerical representation: why seemingly logarithmic representations may rather be multi-linear. Zeitschrift für Psychologie/Journal of Psychology, 219, 64-70.

Moeller, K., Pixner, S., Kaufmann, L., \& Nuerk, H.-C. (2009b). Children's early mental number line: logarithmic or rather decomposed linear? Journal of Experimental Child Psychology, 103, 503-515.

Nuerk, H.-C., Geppert, B. E., van Herten, M., \& Willmes, K. (2002). On the impact of different number representations in the number bisection task. Cortex, 38, 691-715.

Nuerk, H.-C., Moeller, K., Klein, E., Willmes, K., \& Fischer, M. H. (2011). Extending the mental number line: a review of multidigit number processing. Journal of Psychology, 219, 3-22.

Nuerk, H.-C., Weger, U., \& Willmes, K. (2001). Decade breaks in the mental number line? Putting tens and units back into different bins. Cognition, 82, B25-B33.

Opfer, J. E., \& Siegler, R. S. (2007). Representational change and children's numerical estimation. Cognitive Psychology, 55, 169-195.

Price, A. J. (2001). Atomistic and holistic approaches to the early primary mathematics curriculum for addition. In: M. van den Heuvel-Panhuizen (Ed.), Proceedings of the 25th PME International Conference, 4, 73-80.

Rusconi, E., Galfano, G., Speriani, V., \& Umiltà, C. (2004). Capacity and contextual constraints on product activation: evidence from task-irrelevant fact retrieval. Quarterly Journal of Experimental Psychology, 57A, 1485-1511.

Siegler, R. S., \& Booth, J. L. (2004). Development of numerical estimation in young children. Child Development, 75, 428-444.

Siegler, R. S., \& Opfer, J. E. (2003). The development of numerical estimation: evidence for multiple representations of numerical quantity. Psychological Science, 14, 237-243.

Slusser, E., Santiago, R., \& Barth, H. (2013). Developmental change in numerical estimation. Journal of Experimental Psychology: General, 142, 193-208.

Spence, I. (1990). Visual psychophysics of simple graphical elements. Journal of Experimental Psychology: Human Perception and Performance, 16, 683-692. 
Sullivan, J., Juhasz, B., Slattery, T., \& Barth, H. (2011). Adults' number-line estimation strategies: evidence from eye movements. Psychonomic Bulletin and Review, 18, $557-563$.
Wood, G., Nuerk, H.-C., Moeller, K., Geppert, B., Schnitker, R., Weber, J., et al. (2008). All for one but not one for all: how multiple number representations are recruited in one numerical task. Brain Research, 1187, 154-166. 